\title{
Construction of Two-Level Nonregular Designs of Strength Three With Large Run Sizes
}

\author{
Alan R. Vazquez \\ Department of Biosystems, KU Leuven \\ Department of Engineering Management, University of Antwerp \\ and \\ Hongquan $\mathrm{Xu}$ \\ Department of Statistics, University of California, Los Angeles
}

July 25, 2018

\begin{abstract}
Two-level orthogonal arrays of strength 3 permit the study of the main effects and the two-factor interactions of the experimental factors. These arrays are classified into regular and nonregular designs. Good regular designs are available in the literature for large run sizes that are a power of 2 . In contrast, good nonregular designs, which have run sizes that are multiples of 8 and are more flexible alternatives to regular designs, are not available for large numbers of runs because their construction is challenging. In this paper, we introduce a collection of strength-3 nonregular designs with large run sizes that, to the best of our knowledge, have not been explored before in the design literature. Using theoretical results and algorithmic approaches, we construct nonregular designs with up to 1280 runs. Our designs fill the gaps between the available strength-3 designs with large run sizes and outperform many comparably-sized benchmark designs in terms of the aliasing among the two-factor interactions. We show the applicability of our collection of strength-3 designs using an infrared sensor experiment. Supplementary materials for this paper are available online.
\end{abstract}

Keywords: Generalized minimum aberration, generalized resolution, linear permutation, orthogonal array, two-factor interaction, variable neighborhood search. 


\section{Introduction}

Two-level designs are the most commonly used experimental plans for studying the effect of many factors simultaneously. Many two-level designs currently in use involve orthogonal arrays (OAs). Denoting the two levels for each factor by -1 and +1 , these OAs have main effects contrast vectors that are level-balanced and orthogonal to each other.

Due to the rapidly increasing computational power, more and more large two-level OAs are being used in large-scale computer experiments involving simulation of complex physical processes. Most of these processes require the correct identification of the main effects (MEs) and the two-factor interactions (2FIs) of the experimental factors to better understand their performance. This paper is motivated by a practical experiment involving a sensitivity analysis of a surveillance infrared sensor (Lefebvre et al., 2010b). In order to assess the performance of an infrared sensor to detect a simulated combat aircraft, 28 input factors at two levels were considered. The factors included flight conditions variables, aircraft characteristics and atmosphere related factors. The goal of the experiment was to detect the influential effects among the 28 MEs and the 378 2FIs.

A two-level OA has a strength of $t$ if it projects into an equally replicated full $2^{t}$ factorial in every subset of $t$ factors. Consequently, an OA of strength $t$ has a run size that is a multiple of $2^{t}$. The optimal OA for the infrared sensor experiment would have a strength of 4. In this $\mathrm{OA}$, all the $\mathrm{ME}$ contrast vectors and all the $2 \mathrm{FI}$ contrast vectors are orthogonal to each other, and both the ME estimators and the 2FI estimators have a maximum precision. A disadvantage of strength-4 OAs is their run size. For instance, the smallest 28-factor strength-4 OA involves 1024 runs (Hedayat et al., 1999). Lefebvre et al. (2010a) report that a simulation of an infrared sensor and a combat aircraft requires about three minutes. This means that simulating all 1024 two-level combinations of 28 factors in a strength-4 OA would require at least 51 hours. A more economical approach would require an OA of strength 3. Strength-3 OAs have ME contrast vectors that are orthogonal to all 2FI contrast vectors and so, they permit the estimation of all the MEs with full precision, independently from the 2FIs. Although there is correlation between pairs of $2 \mathrm{FI}$ contrast vectors, good strength-3 OAs minimize this correlation and provide attractive cost-efficient alternatives to a strength-4 OA. 
Finding good strength-3 OAs is challenging when the number of factors is larger than 24 or the number of runs is larger than 48 , because it is computationally infeasible to enumerate all OAs for these numbers of factors and runs (Schoen et al., 2010; Bulutoglu and Ryan, 2018). Several methods have been proposed to construct large strength-3 OAs that are regular designs; see Chen et al. (1993), Block and Mee (2005), Lin and Sitter (2008), Xu (2009) and Ryan and Bulutoglu (2010). Regular designs are constructed through defining relations among factors and are described in textbooks such as Mukerjee and Wu (2006) and $\mathrm{Wu}$ and Hamada (2009). These designs have an aliasing structure in which any two interactions are either fully aliased or not aliased at all (orthogonal). A drawback of regular designs is that, if two interactions are fully aliased, it is not possible to estimate both effects at the same time. Another drawback is that they only exist for run sizes that are equal to $2^{u}$, where $u>2$ is an integer. This means that the run size gaps between available options get wider as $u$ increases.

All strength-3 OAs that are not regular designs are called nonregular designs. These designs have run sizes that are multiples of 8 and fill the gaps between the regular designs. In nonregular designs, two interactions may be neither orthogonal nor fully aliased. For this reason, they generally allow more effects to be estimated than comparably-sized regular designs. We refer the reader to Xu (2015) for a recent review on nonregular designs. Unlike regular designs, large nonregular designs are, to a large extent, unexplored research territory. The construction of good nonregular designs is more challenging than the construction of regular designs for two simple reasons: (i) nonregular designs do not have a unified mathematical description and (ii) the class of nonregular designs is much larger than the class of regular designs. Algebraic and combinatorial methods have been proposed to construct large nonregular designs. Xu and Wong (2007) described a systematic procedure for constructing nonregular designs from quaternary linear codes, and constructed designs with up to 256 runs and up to 64 factors. A weakness of their construction method is that it is limited to run sizes that are a power of 2. Mee (2009, ch. 7) provides a comprehensive review of 32-, 36-, 40-, 44- and 48-factor strength-3 nonregular designs constructed by folding-over Hadamard matrices of orders 32, 36, 40, 44 and 48, respectively. Cheng et al. (2008) constructed 192-run strength-3 nonregular designs with 48-60 factors from 
Kronecker products of a Hadamard matrix and a strength-3 OA.

The contribution of this paper is twofold. First, we develop a method to construct large strength-3 nonregular designs for any number of factors and with run sizes that may or may not be a power of 2. Our construction method involves theoretical results and an

algorithmic approach to generate good nonregular designs in terms of the aliasing among interactions. Second, we generate strength-3 nonregular designs with large run sizes that, to the best of our knowledge, have not been explored before in the design literature. We generate a collection of strength-3 nonregular designs with 96-1280 runs and up to 40 factors, for instance for the infrared sensor experiment. Unlike available strength-3 designs, our design options permit the estimation of all the MEs and all the 2FIs for this experiment using a smaller number of runs than a strength-4 OA. Our collection includes many strength-3 nonregular designs that outperform the available benchmark designs in terms of the aliasing among the 2FIs. Our strength-3 nonregular designs whose run sizes are not a power of 2 were previously unknown and fill the gap between available designs of strength 3. An attractive property of our designs is that they can be efficiently partitioned into smaller blocks of runs, thus offering more flexibility for the experimentation. Supplementary materials for this paper include a Matlab implementation of our construction method and supplementary tables to generate our collection of strength-3 designs.

The rest of this paper is organized as follows. Section 2 presents the classification criteria for strength-3 designs. In Section 3, we detail our construction method. Section 4 introduces our large strength-3 nonregular designs and shows how to partition these designs into smaller blocks of runs. We return to the infrared sensor experiment in Section 5 and discuss alternative design options. Section 6 ends the paper with concluding remarks and future research directions.

\section{Classification of strength-3 designs}

We briefly review the $G$-aberration and generalized resolution (Deng and Tang, 1999), and the $G_{2}$-aberration (Tang and Deng, 1999) criteria used to evaluate two-level orthogonal designs of strength 3 . Let $D$ be a two-level design of strength 3 with $N$ runs and $m$ factors. Design $D$ is represented by an $N \times m$ matrix where each row corresponds to a run and 
each column to a factor. Each element of the matrix can take the levels -1 or +1 . For $S=\left\{l_{1}, \ldots, l_{k}\right\}$, a subset of $k$ different factors of $D=\left(d_{i l}\right)$, define

$$
j_{k}(S ; D)=\sum_{i=1}^{N} d_{i l_{1}} \cdots d_{i l_{k}} .
$$

It is easy to see that $j_{k}(S ; D)$ is the sum of the elements of a $k$-factor interaction contrast vector involving the factors in $S$. The $\left|j_{k}(S ; D)\right|$ values are called the $J_{k}$-characteristics. Deng and Tang (2002) showed that the $J_{k}$-characteristics of a strength-3 design $D$ necessarily equal $N-8 q$, where $q \leq N / 8$ is a non-negative integer.

In this paper, we follow Cheng et al. (2004) and use the aliasing index $\rho_{k}(S ; D)=$ $\left|j_{k}(S ; D)\right| / N$ to measure the amount of aliasing between the intercept column and a $k$-factor interaction contrast vector involving the factors in $S$. It is obvious that $0 \leq \rho_{k}(S ; D) \leq 1$. When $\rho_{k}(S ; D)=1$, the $k$-factor interaction involving the factors in $S$ is fully aliased with the intercept, and we say that the factors in $S$ form a complete word of length $k$. When $0<\rho_{k}(S ; D)<1$, the $k$-factor interaction involving the factors in $S$ is partially aliased with the intercept, and we say that the factors in $S$ form a partial word of length $k$ with aliasing index $\rho_{k}(S ; D)$. When $\rho_{k}(S ; D)=0$, the factors in $S$ do not form a word and so the $k$-factor interaction involving the factors in $S$ is not aliased with the intercept. If $D$ is a regular design, a $k$-factor set $S$ either forms a complete word of length $k$ or does not form a word. The $J_{k}$-characteristics and the complete words of a regular design are visible from its defining relation.

For a strength-3 design $D$, any $J_{1^{-}}, J_{2^{-}}$and $J_{3^{-}}$characteristic is zero and thus there are no words of lengths 1, 2 and 3. However, $D$ does have non-zero $J_{4}$-characteristics and partial or complete words of length 4. A 4-factor interaction contrast vector is the Hadamard product of two $2 \mathrm{FI}$ contrast vectors. Whenever a complete word of length 4 occurs in $D$, this implies that 3 pairs of 2 FIs are fully aliased. Partial words of length 4 imply partially aliased 2FIs. If a 4-factor interaction contrast vector is orthogonal to the intercept column, this implies that certain pairs of 2 FIs are not aliased at all. Similarly, words of length 5 account for the aliasing between 2FIs and three-factor interactions (3FIs).

For a set $S$, let $|S|$ be its cardinality. The generalized resolution (Deng and Tang, 1999) 
of a strength- $t$ design $D$ is defined as

$$
G R(D)=r+1-\max _{|S|=r} \rho_{r}(S ; D)
$$

where $r=t+1$. For a strength-3 design $D, 4 \leq G R(D)<5$ and, ideally, the $G R(D)$ value is large because this implies a small maximum $\rho_{4}(S ; D)$ value. If $D$ is a strength-3 regular design, $G R(D)=4$.

The frequencies of the $J_{k}$-characteristics calculated for all $k$-factor interaction contrast vectors are generally collected in a vector $F_{k}=\left(f_{k 1}, \ldots, f_{k v}\right)$, where $v=N / 8$ and $f_{k q}$ denotes the frequency with which the $J_{k}$-characteristics equal $8(v+1-q)$. The frequency of the zero value is usually omitted. The confounding frequency vector (CFV) of a strength3 design $D$ is

$$
C F V(D)=\left[F_{4} ; \ldots ; F_{m}\right]
$$

The G-aberration criterion (Deng and Tang, 1999) sequentially minimizes the components in the CFV from left to right. So, if two designs have $f_{k q}$ as the first nonequal component in their CFVs, the design with smaller $f_{k q}$ is preferred according to the $G$-aberration criterion, as it provides less severe aliasing between pairs of interactions.

A relaxed version of the $G$-aberration criterion is the $G_{2}$-aberration criterion (Tang and Deng, 1999). For $k=1, \ldots, m$, define

$$
B_{k}(D)=\sum_{|S|=k}\left(\rho_{k}(S ; D)\right)^{2}
$$

The vector $\left(B_{1}(D), \ldots, B_{m}(D)\right)$ is called the generalized wordlength pattern (GWLP). For a strength-3 design $D, B_{1}(D)=B_{2}(D)=B_{3}(D)=0$. The $G_{2}$-aberration criterion sequentially minimizes the components of the GWLP from left to right. So, if two designs have $B_{k}$ as the first nonequal component in their GWLPs, the design with smaller $B_{k}$ is preferred according to the $G_{2}$-aberration criterion. If $D$ is regular, both the $\mathrm{CFV}$ and the GWLP reduce to the traditional wordlength pattern (Box and Hunter, 1961). Moreover, the $G$ - and $G_{2}$-aberration criteria are then both equivalent to the traditional aberration criterion (Fries and Hunter, 1980).

Finally, a strength-3 design $D$ allows the estimation of all the MEs with full precision, independently from the 2FIs. Design $D$ is said to be even if $B_{k}(D)=0$ for all odd $k$. In 
an even design, half of the runs are mirror images of the other half, in the sense that the signs of the factor levels are switched. For this reason, it allows at most $N / 2-1$ degrees of freedom for estimating 2FIs (Cheng et al., 2008). Butler (2004, 2007) showed that all strength-3 designs for which $m \geq N / 3$ must be even. If $D$ does not consist of mirror image pairs only, it is called even-odd and has $B_{k}(D)>0$ for some even and odd values of $k$. Even-odd designs generally allow many more degrees of freedom for estimating 2FIs than even designs.

\section{Construction method}

Our construction method concatenates multiple copies of a regular design and performs column permutations as well as sign switches to the elements of one or more columns in the copies. We refer to a copy involving column or row permutations, or sign switches of the elements in one or more columns of the regular design as an isomorphic copy. We use isomorphic copies of a regular design, which we call parent designs, because of their simple aliasing structure that is available from their defining relations. In a regular design, a permutation of the factor columns is equivalent to re-labeling the factors in its defining relation. Moreover, a sign switch of the elements in a factor column is equivalent to a sign change of that factor in the defining relation. If the number of parent designs is not a power of 2, our concatenated design necessarily has a run size that is not a power of 2 . Otherwise, it has a run size that is a power of 2. In either case, the end product of our concatenation procedure is a nonregular design.

Concatenating multiple isomorphic copies of a regular design dates back to John (1961, 1962) and Addelman (1961). These authors concatenated three isomorphic copies involving sign switches of the elements in one or more columns of a regular design to obtain nonregular designs with up to 9 factors and 96 runs. Based on a similar idea, Mee (2004) showed that a 16- and a 47-factor strength-4 designs with 256 and 2048 runs, respectively, can be constructed by concatenating multiple isomorphic copies of a regular design. Pajak and Addelman (1975) considered permuting two columns in copies of a strength-2 regular design and found the minimum number of these copies so that the concatenated design allows the estimation of all the MEs and all the 2FIs. Addelman (1969) and Liao et al. (1996) proposed 
methods to sequentially augment regular designs with isomorphic copies involving only sign switches of the elements in one or more columns. However, these authors' methods use exhaustive enumeration algorithms which are computationally infeasible for constructing concatenated designs with more than 18 factors or 256 runs. In contrast to all these concatenation approaches, our method provides a systematic way to concatenate isomorphic copies of a regular design and permits the construction of nonregular concatenated designs for any number of factors and with more than 256 runs.

We first discuss the effect of column permutations together with the procedure to concatenate several parent designs. Next, we consider sign switches to the elements in one or more columns in the parents so as to improve the concatenated design. For simplicity, we use the terms 'factor permutation' and 'sign switch of a factor' to refer to a permutation of the factor columns and to a sign switch of the elements of a factor column, respectively. We also denote a regular design with $m$ factors and $p$ generators as a $2^{m-p}$ design. The following lemma, central for our construction method, relates the $J_{k}$-characteristics of the parent designs to the ones of the concatenated design:

Lemma 1. The $j_{k}(S ; C)$ value of the concatenated design $C$ can be calculated as the sum of the $j_{k}\left(S ; D_{i}\right)$ values of the parent designs $D_{i}$ over the same $k$-factor set $S$.

The proofs of all the results in this paper are included in Supplementary Section A.

\subsection{Permute all basic factors}

Completely evaluating all possible factor permutations in $d$ copies of a $2^{m-p}$ design is computationally infeasible when $m>10$. For this reason, we choose to limit the evaluation to those from a well-defined subset of permutations, namely the class of linear permutations on the basic factors of the $2^{m-p}$ design. This is because linear permutations have a simple form and allow us to establish general results to concatenate several parent designs. Linear permutations are formally defined as follows:

Definition 1. For an integer $u$, a linear permutation $l_{u}$ over $Z_{b}=\{0, \ldots, b-1\}$ is the permutation such that $l_{u}(x)=x+u(\bmod b)$, for $x \in Z_{b}$. 
Note that there are $b$ linear permutations of $b$ elements and that $l_{0}$ is the identity permutation. For convenience, we also use $Z_{b}=\{1, \ldots, b\}$ when permuting $b$ factors in a $2^{m-p}$ design according to $l_{u}(x)$ so that the new factor labels take values between 1 and $b$.

We permute the basic factors of the $2^{m-p}$ design because this allows us to easily characterize the aliasing structure of the concatenated design. This is illustrated in the following example:

Example 1. Let $D_{0}$ be a $2^{9-4}$ design with basic factors 1, 2, 3, 4 and 5, and generators $6=123,7=124,8=135$ and $9=145$. The defining relation of $D_{0}$ is

$$
\begin{aligned}
I & =1236=1247=1358=1459=3467=2568=234569=234578 \\
& =2579=3489=145678=135679=124689=123789=6789 .
\end{aligned}
$$

A positive or negative word of length $k$ in the defining relation (1) defines a $k$-factor set $S$ with $j_{k}\left(S ; D_{0}\right)$ equal to $+2^{m-p}$ or $-2^{m-p}$, respectively. For instance, 1236 in (1) defines the set $S_{1}=\{1,2,3,6\}$ with $j_{k}\left(S_{1} ; D_{0}\right)=32$. Design $D_{0}$ has a strength of $3, G R\left(D_{0}\right)=4$ and partial GWLP $\left(B_{4}\left(D_{0}\right), B_{5}\left(D_{0}\right), B_{6}\left(D_{0}\right)\right)=(9,0,6)$. Consider an isomorphic copy of this $2^{9-4}$ design, $D_{1}$, formed by applying the linear permutation $l_{1}$ to the set of basic factors. This permutation transforms $1 \rightarrow 2,2 \rightarrow 3,3 \rightarrow 4,4 \rightarrow 5$ and $5 \rightarrow 1$. The defining relation of $D_{1}$ is

$$
\begin{aligned}
I & =2346=2357=1248=1259=4567=1368=134569=134578 \\
& =1379=4589=125678=124679=235689=234789=6789 .
\end{aligned}
$$

Let $D_{2}$ be a second isomorphic copy formed by applying $l_{2}$ to the set of basic factors. That is, $l_{2}$ maps $1 \rightarrow 3,2 \rightarrow 4,3 \rightarrow 5,4 \rightarrow 1$ and $5 \rightarrow 2$. The defining relation of $D_{2}$ is

$$
\begin{aligned}
I & =3456=1347=2358=1239=1567=2468=124569=124578 \\
& =2479=1589=123678=235679=134689=345789=6789 .
\end{aligned}
$$

The defining relations (1), (2) and (3) have one word in common, namely 6789, which contains only the generated factors. Let $C$ be the 9-factor 96-run design constructed by concatenating $D_{0}, D_{1}$ and $D_{2}$. By Lemma 1, the word containing only generated factors defines a complete word of length 4 in $C$ because the factor set $S_{2}=\{6,7,8,9\}$ has 
$j_{4}\left(S_{2} ; C\right)=j_{4}\left(S_{2} ; D_{0}\right)+j_{4}\left(S_{2} ; D_{1}\right)+j_{4}\left(S_{2} ; D_{2}\right)=32+32+32=96$, and so $\rho_{4}\left(S_{2} ; C\right)=$ $96 / 96=1$. This means that the 2 FI pairs 67 and 89,68 and 79 , and 69 and 78 are fully aliased in $C$. Words containing generated and basic factors in (1), (2) and (3) appear in only one defining relation and define partial words of length $k$ with $\rho_{k}(S ; C)=1 / 3$ in $C$, where $k$ equals 4 or 6 . For instance, the word 1236 appears in (1) but not in (2) and (3). So, the factor set $S_{1}$ has $j_{4}\left(S_{1} ; D_{1}\right)=j_{4}\left(S_{1} ; D_{2}\right)=0$. By Lemma $1, j_{4}\left(S_{1} ; C\right)=32$ and thus $\rho_{4}\left(S_{1} ; C\right)=1 / 3$. This implies that the 2FI pairs 12 and 36, 13 and 26, and 16 and 23 are only partially aliased in $C$. The strength-3 9-factor 96-run design $C$ then has 1 complete word of length 4 and 42 partial words; 24 of length 4 with $\rho_{4}(S ; C)=1 / 3$, and 18 of length 6 with $\rho_{6}(S ; C)=1 / 3$. Moreover, $G R(C)=4$ and $\left(B_{4}(C), B_{5}(C), B_{6}(C)\right)=(11 / 3,0,2)$.

Example 1 shows that linear permutations on the set of basic factors of the $2^{m-p}$ design allow a simple characterization of the aliasing structure of the concatenated design. The complete word in the concatenated design contains only the generated factors of the $2^{m-p}$ design. In general, if we permute or re-label the basic factors in the defining relation of a $2^{m-p}$ design, the words that do not change are the ones involving only generated factors, and the ones containing generated factors and all basic factors. These words, if included in the defining relation, result in complete words in a design constructed by concatenating an unmodified copy and isomorphic copies involving linear permutations (or any other class of permutations) on the set of basic factors of the $2^{m-p}$ design. On the other hand, words in the defining relation of the $2^{m-p}$ design that contain generated factors and a subset of basic factors define factor sets that form partial words in the concatenated design. This is because these words are transformed into new ones, which also involve basic and generated factors, in the defining relations of the isomorphic copies as in Example 1. Supplementary Section B shows that permuting other sets of factors such as the generated factors or all factors does not allow such simple characterization of the aliasing structure.

We further simplify the aliasing structure of our concatenated designs by imposing the following condition to the regular designs:

Condition 1. There are no complete words containing generated factors and all basic factors.

This condition ensures that the possible complete words in a concatenated design only 
include the generated factors of the $2^{m-p}$ design used. Note that the $2^{9-4}$ design in Example 1 satisfies Condition 1. We will later show that Condition 1 is not practically relevant for constructing large nonregular designs of strength 3. However, it is useful to establish the following general result to concatenate several isomorphic copies of a $2^{m-p}$ design:

Theorem 1. Consider a $2^{m-p}$ design $D_{0}$ satisfying Condition 1 and let $g$ be its number of complete words containing only generated factors. Let $b=m-p$ be a prime number and $D_{u}$ be the isomorphic copy of $D_{0}$ formed by applying $l_{u}$ to the set of basic factors for $u \neq 0(\bmod b)$. For $d=2, \ldots, b$, the design constructed by concatenating $D_{0}, \ldots, D_{d-1}$ has $2^{m-p} d$ runs, $g$ complete words and $d\left(2^{p}-g-1\right)$ partial words with an aliasing index of $1 / d$.

When $b$ is a prime, the isomorphic copies of $D_{0}$ involving linear permutations on the basic factors have different complete words containing basic and generated factors. Therefore, the $2^{p}-g-1$ complete words containing basic and generated factors in each isomorphic copy result in a total of $d\left(2^{p}-g-1\right)$ partial words with an aliasing index of $1 / d$ in the concatenated design. The lengths of the partial words in Theorem 1 are given by the lengths of the words containing basic and generated factors in the defining relation of $D_{0}$. Similarly, the lengths of the $g$ complete words are given by the lengths of the words containing only generated factors in the defining relation of $D_{0}$. Note that if $D_{0}$ has a strength of 3 , the concatenated design in Theorem 1 has a strength of 3 too and complete and partial words of length at least 4 . Moreover, it is easy to see that if $D_{0}$ is even-odd, Theorem 1 generates a concatenated design that is even-odd too. If $D_{0}$ is even, the concatenated design is also even and provides at most $d\left(2^{m-p-1}-1\right)$ degrees of freedom for estimating 2 FIs. The concatenated design in Theorem 1 has a feature that any interaction involving a subset of basic factors is not fully aliased with any other interactions.

If $b$ is not a prime, the properties in Theorem 1 do not hold because of possibly repeat complete words containing basic and generated factors in the parent designs; see Supplementary Section $\mathrm{C}$ for details. In the next section, we show how to concatenate isomorphic copies of a regular design with a number of basic factors that is not a prime in such a way that the aliasing structure of the concatenated design is still readily available. 


\subsection{Permute a subset of basic factors}

A strategy to concatenate several isomorphic copies of a $2^{m-p}$ design when $m-p$ is not a prime is to permute a prime number of basic factors while fixing the rest. In that case, we apply the permutations $l_{u}$ only to a subset of the basic factors to form the isomorphic copies. For instance, for 6 or 8 basic factors, we permute 5 or 7 basic factors, respectively. For 9 basic factors, we can permute a subset of 5 or 7 basic factors. This strategy results in concatenated designs with complete words of the following types:

Type 1: Those containing only generated factors and the fixed basic factors.

Type 2: Those containing all of the permuted basic factors and other factors.

Type 3: Those containing only generated factors.

On the other hand, each partial word in the concatenated design consists of non-empty subsets of generated factors and permuted basic factors. This is illustrated in the following example:

Example 2. Consider the $2^{10-4}$ design with basic factors 1, 2, 3, 4, 5 and 6 , and generators $7=123,8=1245,9=1246$ and $t_{0}=1356$. Let $D_{0}, D_{1}$ and $D_{2}$ be three isomorphic copies of this $2^{10-4}$ design formed by applying $l_{0}, l_{1}$ and $l_{2}$, respectively, to the subset of basic factors $\{1,2,3,4,5\}$. The defining relations of these designs are

$$
\begin{array}{ll}
D_{0}: \quad & I=1237=12458=12469=1356 t_{0}=34578=34679=2567 t_{0}=5689 \\
& =23468 t_{0}=23459 t_{0}=12356789=14678 t_{0}=14579 t_{0}=1389 t_{0}=2789 t_{0}, \\
D_{1}: & I=2347=12358=23569=1246 t_{0}=14578=45679=1367 t_{0}=1689 \\
& =34568 t_{0}=13459 t_{0}=12346789=25678 t_{0}=12579 t_{0}=2489 t_{0}=3789 t_{0}, \\
D_{2}: \quad & I=3457=12348=13469=2356 t_{0}=12578=15679=2467 t_{0}=2689 \\
& =14568 t_{0}=12459 t_{0}=23456789=13678 t_{0}=12379 t_{0}=3589 t_{0}=4789 t_{0} .
\end{array}
$$

Design $D_{0}$ (as well as $D_{1}$ and $D_{2}$ ) has a strength of $3, G R\left(D_{0}\right)=4$ and partial GWLP $\left(B_{4}\left(D_{0}\right), B_{5}\left(D_{0}\right), B_{6}\left(D_{0}\right), B_{7}\left(D_{0}\right), B_{8}\left(D_{0}\right)\right)=(2,8,4,0,1)$. The defining relations of $D_{0}$, $D_{1}$ and $D_{2}$ do not have words of Types 1, 2 and 3. Consider the 10-factor 192-run design $D$ constructed by concatenating $D_{0}, D_{1}$ and $D_{2}$. By Lemma $1, D$ has 45 partial words 
and no complete words. In particular, it has 6 words with $\rho_{4}(S ; D)=1 / 3,24$ words with $\rho_{5}(S ; D)=1 / 3,12$ words with $\rho_{6}(S ; D)=1 / 3$, and 3 words with $\rho_{8}(S ; D)=1 / 3$. So, $G R(D)=4.66$ and $\left(B_{4}(D), B_{5}(D), B_{6}(D), B_{7}(D), B_{8}(D)\right)=(2 / 3,8 / 3,4 / 3,0,1 / 3)$.

Permuting any other set of 5 basic factors in Example 2 also generates a concatenated design without complete words, except for the sets $\{1,2,3,5,6\}$ and $\{1,3,4,5,6\}$. If the set $\{1,2,3,5,6\}$ is permuted, the concatenated design will have the factor set $\{1,2,3,5,6,7,8,9\}$ as a complete word of Type 2 . If the set $\{1,3,4,5,6\}$ is permuted, the concatenated design will have the factor set $\left\{2,7,8,9, t_{0}\right\}$ as a complete word of Type 1. None of the possible concatenated designs will have words of Type 3.

The following result provides a procedure to concatenate several isomorphic copies of a regular design with a number of basic factors that is not a prime:

Theorem 2. Consider a $2^{m-p}$ design $D_{0}$ and let $B$ be a subset of its basic factors where $b=|B|$ is a prime number. Let $D_{u}$ be the isomorphic copy of $D_{0}$ formed by applying $l_{u}$ to the factors in $B$ for $u \neq 0(\bmod b)$. For $d=2, \ldots, b$, the design constructed by concatenating $D_{0}, \ldots, D_{d-1}$ has $2^{m-p} d$ runs, $h$ complete words and $d\left(2^{p}-h-1\right)$ partial words with an aliasing index of $1 / d$, where $h$ is the number of complete words of Types 1 , 2 and 3 in $D_{0}$.

Although the number of complete words in the concatenated design depends on the set of permuted basic factors, it does not depend on the linear permutations. For this reason, the properties in Theorem 2 hold. The lengths of the $h$ complete words in Theorem 2 are given by the lengths of the complete words of Types 1,2 and 3 in $D_{0}$. Similarly, the lengths of the partial words are given by the lengths of the complete words consisting of non-empty subsets of generated factors and permuted basic factors in $D_{0}$. Theorem 2 does not require $D_{0}$ to satisfy Condition 1 because the words containing generated factors and all basic factors are of Type 2. The concatenated design in Theorem 2 has a feature that any interaction involving a subset of factors in $B$ is not fully aliased with any other interactions.

Finally, for a given number of parent designs, we can construct all concatenated designs by Theorem 2 using all possible subsets $B$ of basic factors. Then, we can choose the best concatenated design in terms of the $G$ - or $G_{2}$-aberration criterion. Clearly, we prefer 
subsets $B$ with large numbers of factors as they reduce the number of possible complete words of Types 1 and 2 in the concatenated design.

\subsection{Algorithmic improvement of designs}

To remove the complete words in our concatenated designs, we apply sign switches to the generated factors in one or more parent designs. This is because, for a $2^{m-p}$ design with possibly permuted factors, sign switches in any set of factors are equivalent to sign switches in a set that only contains generated factors ( $\mathrm{Li}$ and Lin, 2016). Consider the following example:

Example 3. (Continuation of Example 1.) To turn the complete word in the concatenated design $C$ into a partial word, we can change the sign of any generated factor in (1), (2) or (3). For instance, consider $D_{-1}$ to be as $D_{1}$ but with the sign for factor 6 switched. The defining relation of $D_{-1}$ is

$$
\begin{aligned}
I & =-2346=2357=1248=1259=-4567=-1368=-134569=134578 \\
& =1379=4589=-125678=-124679=-235689=234789=-6789 .
\end{aligned}
$$

Let $C^{\prime}$ be the 9-factor 96-run design constructed by concatenating $D_{0}, D_{-1}$ and $D_{2}$. By Lemma 1 , the factor set $S_{2}=\{6,7,8,9\}$ has a $j_{4}\left(S_{2} ; C^{\prime}\right)=j_{4}\left(S_{2} ; D_{0}\right)+j_{4}\left(S_{2} ; D_{-1}\right)+$ $j_{4}\left(S_{2} ; D_{2}\right)=32-32+32=32$. Thus, $\rho_{4}\left(S_{2} ; C^{\prime}\right)=1 / 3$ and $S_{2}$ forms a partial word of length 4 in $C^{\prime}$. This implies that the 2FI pairs 67 and 89, 68 and 79, and 69 and 78, which were fully aliased in $C$, are now only partially aliased. The $J_{k}$-characteristics for sets $S$ containing both basic and generated factors are the same for $C$ and $C^{\prime}$. Therefore, design $C^{\prime}$ has 25 partial words (one more than $C$ ) of length 4 with $\rho_{4}\left(S ; C^{\prime}\right)=1 / 3$ and 18 partial words of length 6 with $\rho_{6}\left(S ; C^{\prime}\right)=1 / 3$. Moreover, $G R\left(C^{\prime}\right)=4.66$ and $\left(B_{4}\left(C^{\prime}\right), B_{5}\left(C^{\prime}\right), B_{6}\left(C^{\prime}\right)\right)=(25 / 9,0,2)$. In contrast, the original design $C$ in Example 1 had $G R(C)=4$ and $\left(B_{4}(C), B_{5}(C), B_{6}(C)\right)=(11 / 3,0,2)$. Hence, $D_{-1}$, obtained by switching the sign of factor 6 , provides a reduction of $8 / 9$ in the $B_{4}$ value and an increase of $2 / 3$ in the generalized resolution of the concatenated design.

The total number of sets of generated factors for which the signs can be switched in $d$ copies of a $2^{m-p}$ design is $2^{p d}$. Evaluating the sign switches in all possible subsets of these 
factors is computationally infeasible when $d>3$ and $p>9$. For this reason, we develop a Variable Neighborhood Search algorithm to search for the best set of generated factors for sign switching in each parent design, so as to minimize the aliasing of our concatenated designs.

Variable Neighborhood Search (VNS, Hansen and Mladenović, 2001) is a metaheuristic that systematically explores more than one neighborhood. A neighborhood is defined by a type of change that can be made to a given solution. The rationale for using more than one neighborhood is that a solution which is a local optimum with respect to one neighborhood is not necessarily a local optimum with respect to another neighborhood. Therefore, escaping from a locally optimal solution can be done by changing the neighborhood. On various occasions, VNS has been used to construct experimental designs; see Garroi et al. (2009), Sartono et al. (2015) and Syafitri and Goos (2015).

Our VNS algorithm performs systematic changes to the parent designs so as to minimize the number of complete words in our concatenated designs. To this end, the algorithm sequentially minimizes the CFV calculated only from the factor sets that define the complete words, because the other words are not affected. Let the isomorphic copies $D_{0}, \ldots, D_{d-1}$ of a $2^{m-p}$ design be formed as in Theorem 1 or 2 , and let $G$ be the set of generated factors appearing in the complete words of the resulting concatenated design. The neighborhood $N_{i}$, $i=1, \ldots, d-1$, involves the sign switch of any one factor in $G$, in $D_{i}$. Note that $|G| \leq p$, thus decreasing the number of factors in which to switch the signs. Our neighborhoods do not include changes in $D_{0}$ since they are irrelevant for the optimization procedure.

Our VNS algorithm is outlined in Algorithm 1. First, a starting concatenated design and the set $G$ are obtained; see lines 1 and 2 in Algorithm 1. The VNS algorithm then explores the first neighborhood $\left(N_{1}\right)$ of the starting design. To this end, it randomly orders the factors in $G$. Next, it selects the first factor in $G$ and an attempt is made to improve the concatenated design by sign switching this factor in $D_{1}$. If a better design is indeed found, the VNS algorithm continues by exploring the first neighborhood of the newly obtained, improved design. If the design does not lead to an improvement, the second factor in $G$ is selected and an attempt is made to improve the initial design by sign switching this factor in $D_{1}$. The exploration of $N_{1}$ continues until the sign switch of every single factor in $G$ has 


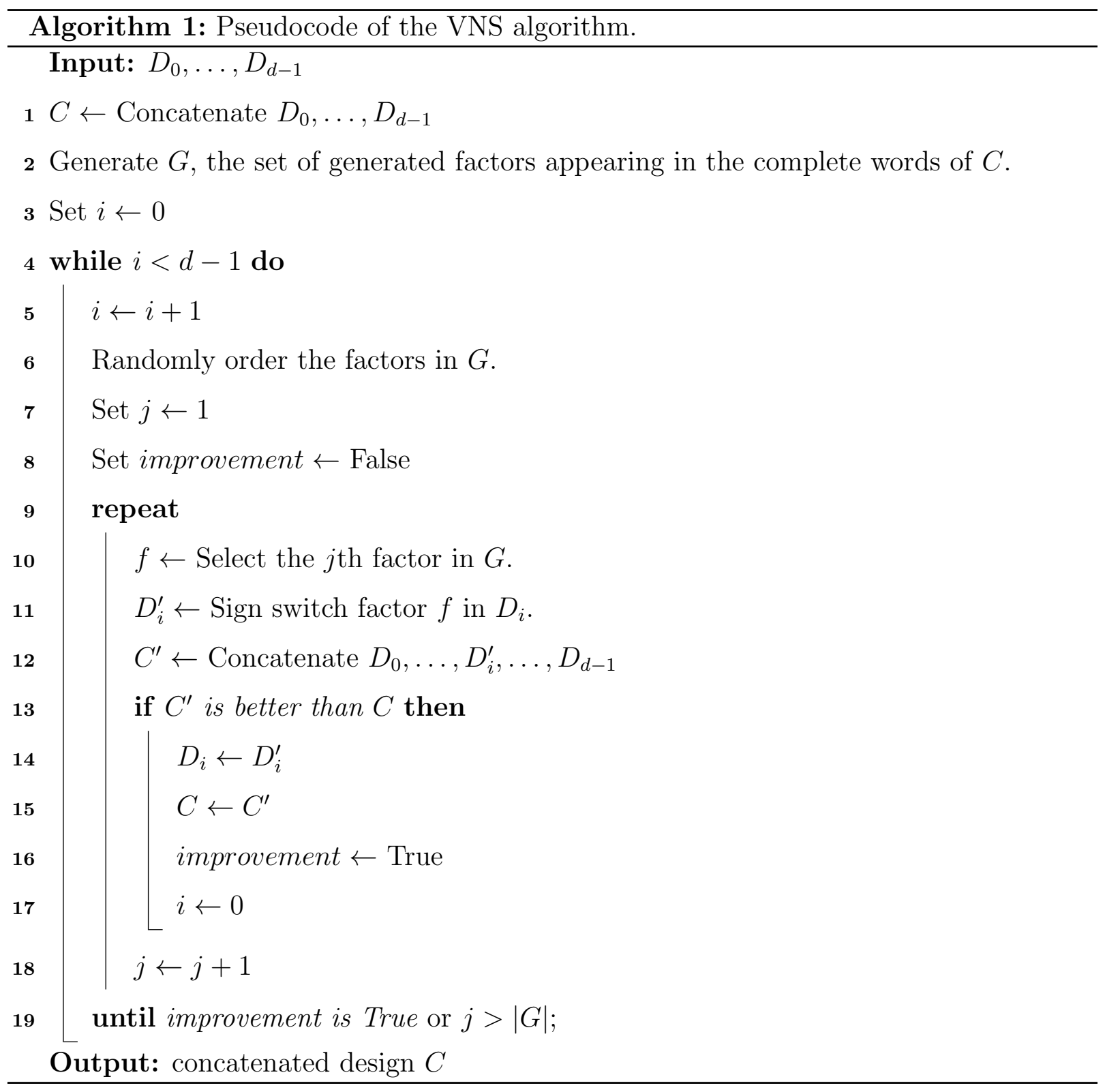

been tested in $D_{1}$. If this does not yield any better concatenated design than the current best one, the algorithm starts exploring the second neighborhood $\left(N_{2}\right)$, in the same fashion. As soon as the exploration of $N_{2}$ results in an improved design, the VNS algorithm returns to $N_{1}$ and explores that first neighborhood of the improved design. If the exploration of the second neighborhood does not produce an improved design, the third neighborhood $\left(N_{3}\right)$ is explored, and so on, until the last neighborhood $\left(N_{d-1}\right)$ is explored. The process is repeated until no further improvement can be reached.

To increase the likelihood of finding the best possible improved design, the VNS algo- 
rithm is repeated a number of times, each time exploring the factors in $G$ in a random order in each parent design, and the overall best design is reported. We include a Matlab implementation of the VNS algorithm in the supplementary materials.

\section{Large nonregular designs of strength three}

We construct two-level nonregular designs of strength 3 with 96-1280 runs and up to 40 factors. Our designs minimize the number of complete and partial words of length 4 because they quantify the most serious aliasing in strength-3 designs, namely the aliasing among the 2FIs. We first detail the regular designs used and the specifications for our construction procedure. Next, we discuss the collection of strength-3 designs and show how to partition these designs into smaller blocks of runs.

\subsection{Specifications}

We use strength-3 regular designs that have minimum aberration (MA). More specifically, we consider MA designs with 32, 64, 128 and 256 runs and 5, 6, 7 and 8 basic factors, respectively. The 32-run MA designs with 11 factors or more and the 64-run designs with

21 factors or more are even. The rest of our regular designs are even-odd. Details regarding these designs are included in Supplementary Section D.

We construct concatenated designs using Theorems 1 and 2 with $d=3,4$ and 5 for the 32-, 64- and 256-run MA designs. For the 128-run MA designs, we consider $d=3, \ldots, 7$. A close inspection of the MA designs with a prime number of basic factors revealed that some of them have complete words containing generated factors and all basic factors. So, they do not satisfy Condition 1 in Theorem 1. However, these words necessarily have a length larger than 5 and, consequently, they do not affect the aliasing among the 2FIs. For this reason, we can ignore Condition 1 when constructing strength-3 concatenated designs from MA designs with 5 and 7 basic factors.

For the 64-run MA designs, we consider subsets of basic factors $B$ with $|B|=5$ in Theorem 2. We improve all six concatenated designs using the VNS algorithm and report the best design and the best factor to keep fixed. We construct concatenated designs from 
the 256-run MA designs in a similar fashion using subsets of basic factors $B$ with $|B|=7$. The concatenated designs from the 64- and 256-run MA designs do not have complete words of length 4 of Type 2 as the number of permuted basic factors is 5 and 7 , respectively.

To improve our concatenated designs, we consider the $F_{4}$ vector as the objective function for the VNS algorithm because we favor designs with the smallest number of complete words of length 4 . Note that sequentially minimizing the $F_{4}$ vector automatically maximizes the generalized resolution. For each design case, we execute the VNS algorithm 100 times. As the best solution is usually found after only a few iterations, we consider 100 to be more than enough. Detailed analysis of the operation of the algorithm showed that all neighborhoods are used when searching for the best solution. The computing times of the algorithm are between 11 and 17 seconds per iteration on a standard CPU (Intel(R) Core(TM) i7 processor, $2.8 \mathrm{Ghz}, 8$ GB) to optimize a 40-factor 768-run design constructed from 6 isomorphic copies of the MA $2^{40-33}$ design. In Supplementary Section E, we highlight the contributions of our theoretical results and the VNS algorithm for constructing nonregular strength-3 designs.

\subsection{Tables of designs}

Tables 1-4 present the most interesting strength-3 designs we found. Our complete collection of designs, the factors for which to switch the signs in the parent designs, and the best basic factors to keep fixed in the 64- and 256-run MA designs are included in Supplementary Sections F and G. Tables 1-4 include the number of factors, the number of runs, the generalized resolution (GR), the $F_{4}$ vector, the $B_{4}$ value and the number of parent designs $(d)$ of our concatenated designs.

\subsubsection{Designs obtained from 32-run regular designs}

Table 1 shows strength-3 designs with 96-160 runs and 9-16 factors obtained from the 32-run MA designs. The 96-run designs have a GR value of 4.66, except for the 15- and 16-factor designs which have a GR value of 4. For 9, 10 and 11 factors, in principle, 96 runs is enough to estimate all the 2FIs in addition to the MEs. However, only our 96-run designs with 9 and 10 factors permit the estimation of all these effects simultaneously. Our 
96-run designs with 11 factors or more do not allow the estimation of the 2FIs because they are constructed from three isomorphic copies of an even design. Thus, they do not allow the estimation of more than $3(32 / 2-1)=45$ 2FIs. For 9, 10 and 11 factors, alternative 96-run designs of strength 2 that do allow the estimation of all the MEs and all the 2FIs simultaneously can be found in Mee (2009, ch. 8). A drawback of these designs is that the ME contrast vectors are correlated with the $2 \mathrm{FI}$ contrast vectors.

Table 1 shows 160-run designs with 10-16 factors and a 128-run design with 16 factors. The 160-run designs have a GR value of 4.8, except for the 15- and 16-factor designs which have a GR value of 4.4. The 16-factor 128-run design has a GR value of 4.5. For 128 runs, benchmark 16-factor designs of strength 3 are available in Xu and Wong (2007) and $\mathrm{Xu}$ (2009). Our 128-run design outperforms the benchmark designs in terms of the $G$ aberration criterion. Note that the 16-factor 128-run design in Table 1 has a larger GR value than the corresponding design with 160 runs. However, the 160-run design has a better $B_{4}$ value than the smaller alternative.

Table 1 also shows that the 160-run designs with 10, 11 and 12 factors permit the estimation of all the 2FIs. For 13 factors or more, our 160-run designs do not allow the estimation of all the 2FIs because they are constructed from five isomorphic copies of an even design. So, they cannot estimate more than $5(32 / 2-1)=75$ 2FIs.

Table 1 does not report our 128-run designs with 10-15 factors nor our 9-factor 160-run design because comparable designs of strength 4 are available in the literature (Hedayat et al., 1999; Bulutoglu and Ryan, 2018). Although smaller strength-4 alternatives to our 160-run designs with 10-15 factors exist, the table does report our strength-3 160-run designs since, for these numbers of factors, strength-4 160-run designs do not exist; see Bulutoglu and Ryan (2018). All our strength-3 designs with 128 and 160 runs are shown in Supplementary Section F.

A referee pointed out that some of our concatenated designs have repeat runs. More specifically, the 10-factor 96-run design and the 160-run designs with 12-14 factors in Table 1 have two duplicate runs each. Other designs with repeat runs in the table are the 9-factor 96-run design and the 11-factor 160-run design with four duplicate runs each, and the 10-factor 160-run design with two quadruplicate runs. Repeat runs are attractive for 
physical experiments as they provide a pure error estimate of the error variance. However, repeat runs are not helpful for computer experiments where the simulations are deterministic. Supplementary Section F shows that 36 out of all the 193 concatenated designs we constructed have repeat runs.

\subsubsection{Designs obtained from 64-run regular designs}

Table 2 shows strength-3 designs with 192-320 runs and 17-26 factors obtained from the 64-run MA designs. For 21 factors or more, the designs in the table are even since their 64-run parent designs are even; see Supplementary Section D for details on these parents.

Table 2 shows that our 192-run designs with 17 and 18 factors have a GR value of 4.66. For 19 factors or more, our 192-run designs have a GR value of 4. For 20-26 factors, the table shows strength-3 designs with 256 runs. Our 256-run designs have a GR value of 4, except for 20 and 21 factors. For these numbers of factors, the 256-run designs have a GR value as large as 4.5. Strength-3 benchmarks with 256 runs are available in Xu and Wong (2007) and $\mathrm{Xu}$ (2009). Our 256-run designs outperform the best regular designs in $\mathrm{Xu}$ (2009) in terms of the $G$-aberration criterion. For 20 and 21 factors, our 256-run designs also have a smaller $G$-aberration than the benchmarks obtained from quaternary linear codes (QLCs) in Xu and Wong (2007). However, for 22 factors or more, the QLC designs have a GR value as large as 4.5 and thus a smaller $G$-aberration than our 256-run designs. The fact that the 256-run QLC designs have larger GR values than our designs is because they are even-odd. This means that they cannot be constructed by concatenating four strength-3 64-run designs, which are even for 22 factors or more. Therefore, the 256-run QLC designs necessarily consist of four 64-run designs of strength 2 or less.

Table 2 gives 320-run designs with 20-26 factors and a GR value of 4.4. For 20 and 21 factors, these designs provide a smaller GR value but a better $B_{4}$ value than the 256-run designs in the table. It is interesting to mention that for 24 factors or more, our 320-run designs provide a larger GR value than the regular 512-run designs in $\mathrm{Xu}$ (2009), which have a GR value of only 4. So, in contrast with the regular 512-run designs, the smaller designs do not have pairs of 2FIs that are completely aliased. For 17-19 factors, Table 2 does not report our strength-3 designs with 256 and 320 runs because strength-4 256-run 
designs for these numbers of factors are available in Hedayat et al. (1999).

\subsubsection{Designs obtained from 128-run regular designs}

Table 3 shows strength-3 designs with 384-896 runs and 20-40 factors obtained from the 128-run MA designs. To shorten the discussion, the table includes only our strength-3 designs with a GR value larger than 4. For some numbers of factors, the table omits some design options because alternative options with a smaller run size and a larger GR value are available; see Supplementary Section F for details. Table 3 also does not include the designs with 20-23 factors and 512 runs or more because strength-4 512-run designs for these numbers of factors are available in $\mathrm{Xu}(2009)$.

Table 3 shows 384-run designs with 20-25 factors and a GR value of 4.66. For 20-22 factors, these designs permit the estimation of all the 2FIs. The table also shows 512-run designs for 24 and 25 factors with a GR value as large as 4.75. For 26-29 factors, our 512-run designs have a GR value of 4.5. The 512-run designs in the table outperform the strength-3 regular designs in Xu (2009) in terms of the GR value and the $G$-aberration criterion. Supplementary Section F includes more 512-run designs with a GR value of 4 that outperform the best regular designs in $\mathrm{Xu}$ (2009) in terms of the $G$-aberration criterion. Table 3 shows that only our 512-run designs with 24 and 25 factors permit the estimation of all the 2FIs.

It is interesting to point out that, in contrast to the regular 512-run designs with 24 and 25 factors in $\mathrm{Xu}$ (2009), which have a GR value of 4, our 384-run designs with the same number of factors do not involve complete words of length 4 and therefore have a GR value larger than 4; see Table 3. Consequently, they are attractive cost-efficient alternatives to the regular 512-run designs.

Table 3 includes 640-run designs with 24-26 and 30-36 factors. The designs with 24-26 factors have a GR value of 4.8 and allow the estimation of all the 2FIs. The 640-run designs with 27-36 factors have a GR value of 4.4. The table lists 768-run designs with 27-35 and 37-40 factors, which have GR values of 4.66 and 4.33, respectively. Table 3 shows 896-run designs with 24-29 factors and a GR value as large as 4.857. For 36 and 37 factors, the table also includes 896-run designs with a GR value of 4.571. For 24-29 factors, the designs 
with 768 and 896 runs permit the estimation of all the 2FIs.

For 34-40 factors, Table 3 includes 640- and 768-run design options with a better aliasing for the 2FIs than the best 1024-run regular designs in $\mathrm{Xu}$ (2009). This is because the pairs of 2FIs are at most partially aliased in our designs. In contrast, the 1024-run benchmarks have a GR value of 4 and thus have pairs of 2 FIs that are completely aliased.

From the designs in Table 3, only the 24-factor designs with 640 and 896 runs, and the 25-factor 896-run design have repeat runs. More specifically, they have two duplicate runs each.

\subsubsection{Designs obtained from 256-run regular designs}

Table 4 shows strength-3 designs with 768-1280 runs and 24-36 factors obtained from the 256-run MA designs. For 24-33 factors, the table does not report our strength-3 designs with 1024 and 1280 runs since 1024-run strength-4 designs are available in Xu (2009). Our 1024- and 1280-run strength-3 designs for these numbers of factors are included in Supplementary Section F.

For 768 runs, Table 4 shows that our designs with 24-34 factors have a GR value of 4.66, whereas our designs with 35 and 36 factors have a GR value of 4. The 34-factor 1024-run design in the table has a GR value as large as 4.75. For 35 and 36 factors, our 1024-run designs have a GR value of 4.5. The 1024-run options in the table outperform the best regular designs in $\mathrm{Xu}$ (2009) in terms of the $\mathrm{GR}$ value and the $G$-aberration criterion. It is also interesting to point out that our 34-factor 768-run design has a larger GR value than the best 1024-run regular design in Xu (2009), which has a GR value of only 4.

Table 4 shows that our 1280-run designs have a GR value of 4.8 and permit the estimation of all the 2FIs. Other designs that allow the estimation of these effects simultaneously are the 768-run designs with 24-29 factors and all the 1024-run designs.

As shown in Table 3, for 24-26 factors, our 512- and 640-run designs constructed from four and five 128-run parent designs, respectively, have larger GR values than the 768run alternatives constructed from three 256-run parents in Table 4 . This is because the maximum GR value for a design constructed from three parents is 4.66. In contrast, a design constructed from four or five parents can have a GR value as large as 4.75 and 4.8, 
respectively. For 27-34 factors, the 768-run designs in Table 3 have a smaller $G$-aberration than the 768-run designs in Table 4. However, the 768-run designs with 24-34 factors in Table 4 have a smaller $B_{4}$ value than the 512-, 640- and 768-run designs with the same numbers of factors constructed from 128-run MA designs.

Finally, only the 24-factor 768-run design in Table 4 has repeat runs. More specifically, this design has four duplicate runs.

\subsection{Blocking}

For some situations, using our strength-3 designs for a completely randomized experiment may be infeasible because of their large run sizes. A more convenient approach may be to partition the designs into smaller blocks of runs that can be conducted on different machines or days. An attractive property of our concatenated designs is that they can easily be partitioned in orthogonal blocks. This can be done using each parent design as a block:

Lemma 2. A design constructed by concatenating $d$-run designs of strength $t \geq 2$ can be arranged into $d$ blocks of size $n$ such that the block effects are not confounded with the main effects and all the $j$-factor interactions for $j=2, \ldots, t$.

Lemma 2 implies that the strength-3 designs in Tables 1-4, and also all the strength-3 designs in Supplementary Section F, can be arranged into $d$ blocks of size $n$, with $d$ the number of parent designs and $n$ the run size of the regular design used, so that the block effects are not confounded with the MEs, the 2FIs and even the 3FIs. For instance, the 28-factor 768-run design in Table 4 can be split into 3 blocks of size 256. Assuming that block and treatment interactions are negligible, differences between the blocks of 256 runs will not bias the estimation of the MEs and the 2FIs.

Let $c$ be a divisor of $d$. From Lemma 2, it is evident that our concatenated designs constructed using a number of $n$-run parent designs $d$ that is not a prime, can also be partitioned into $c$ blocks of size $n d / c$ so that the MEs, the 2FIs and the 3FIs are not confounded with the block effects. For instance, the 28-factor 768-run design in Table 3, constructed from six 128-run parents, can be arranged into 2, 3 or 6 blocks of sizes 384 , 256 and 128, respectively, with the aforementioned properties. 


\section{The infrared sensor experiment}

The practical experiment that motivated this paper involves a sensitivity analysis of a surveillance infrared sensor (Lefebvre et al., 2010b). In order to assess the performance of an infrared sensor to detect a simulated combat aircraft, 28 input factors at two levels were considered. The goal of the experiment was to estimate the two-factor interaction model, that is, the model containing the intercept, the $28 \mathrm{MEs}$ and the $3782 \mathrm{FIs}$, and to detect the influential effects. It was assumed that three-factor and higher order interactions were negligible. The design actually used was a strength-4 OA with 1024 runs that provided full precision to estimate all the effects of interest.

The work we did in this paper allowed us to find three cost-efficient alternatives for the infrared sensor experiment. Table 5 contains 768- and 896-run nonregular strength-3 designs that can estimate the two-factor interaction model in 28 factors. The table shows the generalized resolution (GR), the $F_{4}$ vector, the $B_{4}$ value and the D-efficiency (shown as a percentage) for the two-factor interaction model of the design options. The D-efficiency of a design $D$ with an $N \times p$ model matrix $X_{M}$ is defined as $\left(\operatorname{det}\left(X_{M}^{T} X_{M}\right)\right)^{1 / p} / N$. The 768-run designs can be partitioned into 3 or 6 blocks of 256 or 128 runs, respectively. The 896-run design can be split into 7 blocks of 128 runs.

For all designs in Table 5, the MEs can be estimated with full precision and are not aliased with the 2FIs. Regarding the aliasing among the 2FIs, both 768-run designs have a generalized resolution as large as 4.66. The 768-run design constructed from 6 blocks of size 128 has $25 J_{4}$-characteristics of 256 and thus 75 pairs of $2 \mathrm{FIs}$ that are partially aliased with an aliasing index of only 0.33 . This design has other 2610 pairs of 2 FIs that are partially aliased with an aliasing index of 0.16. On the other hand, the 768-run design constructed from 3 blocks of size 256 has $152 J_{4}$-characteristics of 256 and thus 456 pairs of $2 \mathrm{FIs}$ with an aliasing index of 0.33 ; 381 pairs more than the design constructed from blocks of size 128. The 896-run design has a generalized resolution of 4.857. So, each pair of 2 FIs in this design has an aliasing index of either 0.143 or 0 .

Among the design options in Table 5, the 768-run design constructed from 3 blocks of size 256 has the smallest $B_{4}$ value. Cheng et al. (2002) established an approximate relationship between the D-efficiency for the two-factor interaction model and the $B_{4}$ value 
of a strength-3 design. According to their relationship, a strength-3 design with a small $B_{4}$ value tends to have a large D-efficiency. For this reason, the 768-run design constructed from 3 blocks of size 256 has the largest D-efficiency for the two-factor interaction model. The fact that the D-efficiencies in Table 5 are between $74 \%$ and $84 \%$ is because we restrict ourselves to orthogonal designs that have ME contrast vectors that are orthogonal to the 2FI contrast vectors.

Lefebvre et al. (2010a) report that a simulation of an infrared sensor and a combat aircraft requires about three minutes. Therefore, simulating all two-level combinations of our strength-3 designs with 768 and 896 runs would require around 38 and 44 hours, respectively, instead of 51 hours that required with the strength-4 1024-run design. This would have saved 13 and 7 hours of computing time using the 768- and 896-run design options, respectively. Further computing time savings can be achieved by running the blocks of runs of the designs in parallel on different machines. The machines may have different components such as numbers of processors, memory capacities and random generators, which may result in different precisions and round-off errors for the simulations; see He and Ding (2001), Click et al. (2011) and Harvey and Verseghy (2016) for studies showing how different round-off errors and random generators may produce different numerical simulations of complex processes. In our design options, block effects associated to the machines would not be confounded with the MEs nor with the 2FIs. For the infrared sensor experiment, we would prefer the 768-run design constructed from 3 blocks of size 256 because of its run size economy and its higher D-efficiency for the two-factor interaction model in 28 factors.

An alternative approach for the infrared sensor experiment that departs from OAs is the optimal design approach (Atkinson et al., 2007). Using the coordinate-exchange algorithm (Meyer and Nachtsheim, 1995), as implemented in JMP 13, we constructed completely randomized designs with 768 and 896 runs that maximize the D-efficiency for the twofactor interaction model in 28 factors. We also constructed a 768-run block randomized design in 6 blocks of size 128, a 768-run block randomized design in 3 blocks of size 256, and a 896-run block randomized design in 7 blocks of size 128. Assuming a model including the intercept, the block effects, all the MEs and all the 2FIs, these block randomized designs maximize the D-efficiency for estimating the factors' effects. For each design problem, we 
specified a maximum search time of 2 hours in JMP 13. We refer to the designs found by the coordinate-exchange algorithm as D-efficient, since there is no guarantee that these designs achieve the maximum possible D-efficiency.

The D-efficient completely randomized designs were not orthogonal and had every pair of effects partially aliased. The D-efficiencies of the 768- and 896-run designs for the twofactor interaction model in 28 factors were $89.03 \%$ and $91.81 \%$, respectively. Figure 1 compares the performance of these D-efficient designs to our strength-3 designs in terms of the standard errors for the ME and the 2FI estimates. The figure shows that the strength-3 designs outperform the D-efficient designs in terms of the estimation of the MEs as they provide the smallest standard errors (SEs). Figure 1 shows that the maximum SE for the 2FI estimates is around 0.078 for the 768-run strength-3 designs. However, the median SE for the 2FI estimates is different for these designs; the median SE for the design constructed from 6 blocks of size 128 is around 0.054 whereas for the design constructed from 3 blocks of size 256 is around 0.045. For the 896-run strength-3 design, the maximum and median SEs for the 2FI estimates are 0.062 and 0.045, respectively. Figure 1 shows that the SEs for the 2FI estimates based on the D-efficient designs are much more homogeneous and also have a smaller maximum than the corresponding strength-3 designs.

The D-efficient block randomized designs provided block effects that were partially confounded with the MEs and the 2FIs. Moreover, the ME contrast vectors were not orthogonal to the $2 \mathrm{FI}$ contrast vectors in the D-efficient block designs. A comparison (not shown here) between the D-efficient block designs and our blocked strength-3 designs revealed similar results for the standard errors of the ME and the 2FI estimates as for the completely randomized designs.

Our comparisons between the D-efficient designs and the strength-3 designs assume that the MEs and the $2 \mathrm{FIs}$ are equally important. In that case, the D-efficient designs are attractive alternatives. On the other hand, if the MEs are considered to be more important than the 2FIs or, in other words, that effect hierarchy holds (Wu and Hamada, 2009, ch. 4), the strength-3 designs are more attractive as they can estimate all the MEs with full precision while providing good detectability for 2FIs. More comparisons between the performances of OAs and the optimal design approach can be found in Schoen (2010) 


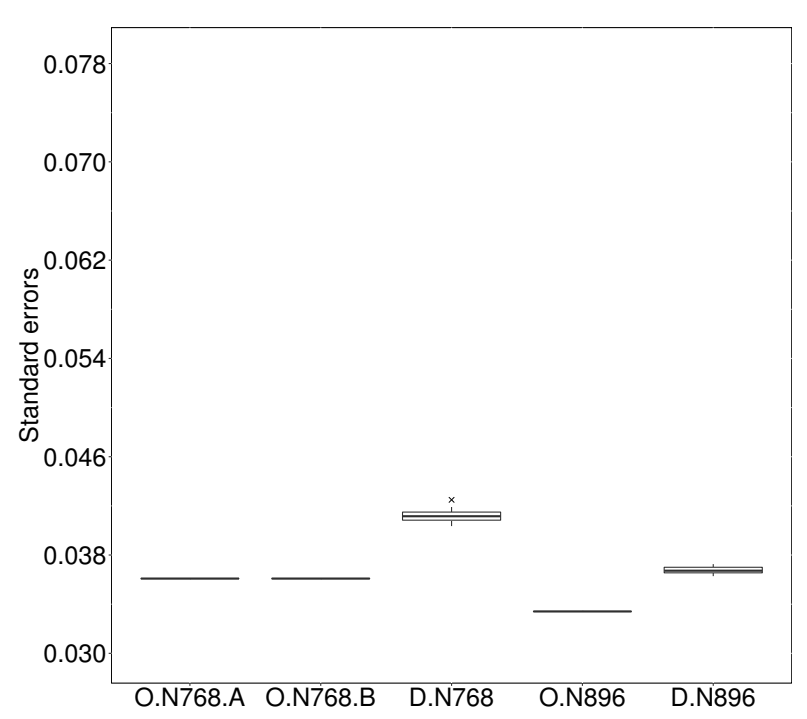

(a) Main effects

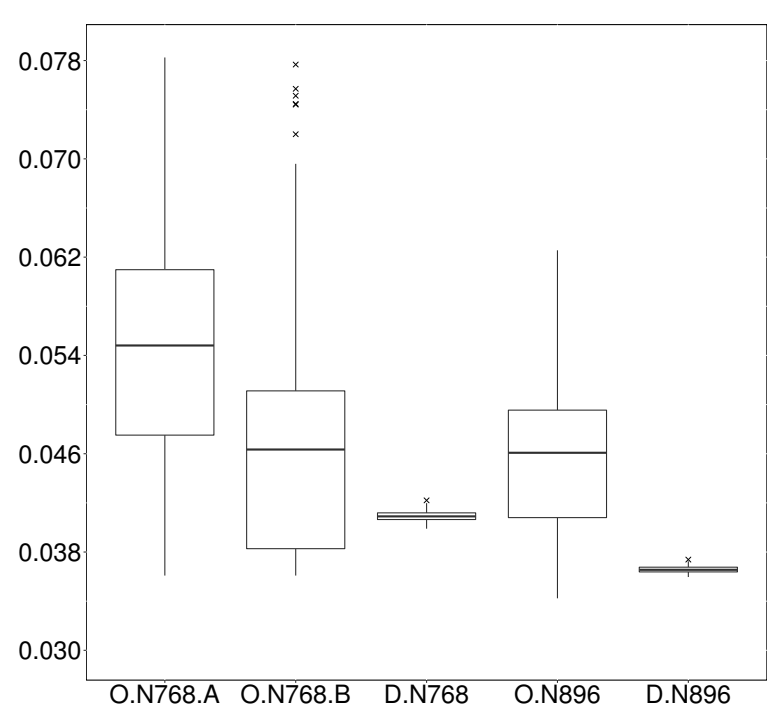

(b) Two-factor interactions

Figure 1: Standard errors for effect estimates in 28-factor designs with 768 and 896 runs. O: OA of strength 3; D: D-efficient design; O.N768.A: strength-3 OA constructed from 6 blocks of size 128; O.N768.B: strength-3 OA constructed from 3 blocks of size 256.

and Eendebak and Schoen (2017).

Finally, our blocked designs have some unique features that the D-efficient block designs do not have. When running the simulations in blocks on multiple machines, each block provides an estimate of the MEs. Because the block effects are not confounded with the MEs and the 2FIs in our blocked designs, one can use the estimates of the MEs from different blocks or machines to cross validate the results and to detect possible inconsistencies or dependencies of the machines. Moreover, in the event that one of the machines produces undesirable results, the reduced design would still have a strength of 3 and so it would still be possible to estimate the MEs with full precision, independently from the 2FIs and the block effects.

\section{Concluding remarks}

In this paper, we constructed large two-level nonregular designs of strength 3 . We proposed a method to construct the strength-3 designs by concatenating several isomorphic copies of 
a smaller two-level regular design with the same strength. Our construction method relies on theoretical results to concatenate the isomorphic copies and an algorithmic approach to improve the concatenated design. Our theoretical results suggest a systematic way in which to permute a subset of factors in the copies and generate strength-3 designs with an aliasing structure in which most, if not all, pairs of $2 \mathrm{FIs}$ are either partially aliased or not aliased at all. The partially aliased interactions have an aliasing degree inversely proportional to the number of concatenated copies. A set of complete words, consisting only of the factors that are not permuted, may be present in our nonregular designs depending on the defining relation of the regular design used. However, our VNS algorithm eliminates the complete words from this set by sign switching one or more generated factors in the parent designs. The supplementary materials include a Matlab implementation of our construction method.

We generated strength-3 nonregular designs with large run sizes that, to the best of our knowledge, have not been explored before in the design literature. We constructed nonregular strength-3 designs with 96-1280 runs and 9-40 factors. These designs were generated from minimum aberration regular designs of resolution 4 with 32, 64, 128 and 256 runs. Our nonregular designs with run sizes that are not a power of 2 were previously unknown and fill the gaps between available strength-3 designs with large run sizes. Our nonregular designs with run sizes that are a power of 2 outperform the regular strength3 designs of $\mathrm{Xu}$ (2009) and some nonregular strength-3 designs of Xu and Wong (2007), in terms of the aliasing among 2FIs. Supplementary Section G shows how to generate our collection of strength-3 nonregular designs using supplementary tables. An attractive property of our strength-3 designs is that they can be easily blocked so that the block effects are not confounded with the MEs and the 2FIs. We showed the usefulness of our strength-3 designs using an infrared sensor experiment.

Our constructed strength-3 designs are also useful for large-scale physical experiments. Take the drug combination experiment as an example. Multi-drug combinations, which deliver better efficacy and reduced side effects compared to single drug regimens, are applied to treat malignant diseases such as cancers (Devita et al., 1975; Straetemans et al., 2005; Chou, 2006; Ning et al., 2014). During the preparation of this paper, one of the authors was called by his colleagues to help design a cancer immunotherapy experiment involving 
21 drugs. The researchers could afford a maximum of 477 two-level combinations indicating the presence or absence of the drugs. The 384-run design in Table 3 is a good choice as it can estimate all the MEs and all the 2FIs. As the design has a generalized resolution of 4.66, the MEs can be estimated with maximum precision, which is preferred by the researchers in order to screen important drugs. The researchers are currently testing drug combinations using this design.

The data analysis for the strength-3 designs that permits the estimation of the twofactor interaction model may be conducted using linear regression. Note that if the design has repeat runs, these may be used to estimate the error variance for the significance tests. For the designs that do not permit the estimation of the two-factor interaction model, one may conduct the two-stage analysis of Miller and Sitter (2001). In the first step, standard significance tests are used to identify the active MEs. Such tests can also benefit from a pure error estimate of the error variance given by repeat runs in the design. In the second stage, model selection techniques are used to identify the active 2FIs among those factors identified in first stage, assuming that the number of active 2FIs is relatively small.

For 128, 256, 512 and 1024 runs, we did not find better designs than the best regular strength-3 designs in terms of the $G_{2}$-aberration criterion, because our focus on minimizing the $G$-aberration criterion. An interesting topic for future research is to develop methods for concatenating regular designs, provably of strength 2 or 3 , so that the resulting designs outperform the best regular designs in terms of the $G_{2}$-aberration criterion. Good designs can also be constructed by concatenating nonregular designs. For instance, concatenating isomorphic copies of a nonregular design with a larger generalized resolution than a regular design may lead to better concatenated designs than ours, in terms of the $G$-aberration criterion. However, a challenge in the concatenation of nonregular designs is their complex aliasing structure. Recently, Mee (2017) showed that the aliasing structure of some nonregular designs can be summarized using defining relations. Another interesting topic for future research is to concatenate isomorphic copies of these nonregular designs and to study the aliasing structure of the concatenated designs through the defining relations. To this end, we may also consider other classes of permutations such as the exponential or logarithmic permutations used in Xiao and Xu (2017). 


\section{SUPPLEMENTARY MATERIALS}

Supplementary sections.pdf Proofs; issues with permuting other sets of factors and with $2^{m-p}$ designs with $m-p$ not a prime number; details of regular designs used; contributions of our theorems and algorithm to construct nonregular designs; tables of two-level strength-3 designs; and, sets of factors for sign switching in the parent designs.

Programs.zip Matlab implementation of the VNS algorithm.

\section{ACKNOWLEDGEMENTS}

Vazquez was financially supported by the Flemish Fund for Scientific Research FWO and $\mathrm{Xu}$ was supported by NSF Grant DMS-1407560. The research was done while the first author was visiting the Department of Statistics at the University of California, Los Angeles. The authors thank Peter Goos, Eric Schoen and three referees for their comments that help to improve the presentation of the paper. The authors also thank Sidonie Lefebvre for sharing details about the infrared sensor experiment.

\section{References}

Addelman, S. (1961). Irregular fractions of the $2^{n}$ factorial experiments. Technometrics, 3:479-496.

Addelman, S. (1969). Sequences of two-level fractional factorial plans. Technometrics, 11:477-509.

Atkinson, A. C., Donev, A. N., and Tobias, R. D. (2007). Optimum Experimental Designs, With SAS. Oxford University Press.

Block, R. M. and Mee, R. W. (2005). Resolution IV designs with 128 runs. Journal of Quality Technology, 37:282-293. 
Box, G. and Hunter, J. (1961). The $2^{k-p}$ fractional factorial designs. Technometrics, 3:311-351, 449-458.

Bulutoglu, D. A. and Ryan, K. J. (2018). Integer programming for classifying orthogonal arrays. Australasian Journal of Combinatorics, 70:362-385.

Butler, N. A. (2004). Minimum $G_{2}$-aberration properties of two-level foldover designs. Statistics 8 Probability Letters, 67:121-132.

Butler, N. A. (2007). Results for two-level fractional factorial designs of resolution IV or more. Journal of Statististical Planning and Inference, 137:317-323.

Chen, J., Sun, D. X., and Wu, C. F. J. (1993). A catalogue of two-level and three-level fractional factorial designs with small runs. International Statistical Review, 61:131-145.

Cheng, C.-S., Deng, L.-Y., and Tang, B. (2002). Generalized minimum aberration and design efficiency for nonregular fractional factorial designs. Statistica Sinica, 12:9911000.

Cheng, C.-S., Mee, R. W., and Yee, O. (2008). Second order saturated orthogonal arrays of strength three. Statistica Sinica, 18:105-119.

Cheng, S.-W., Li, W., and Ye, K. Q. (2004). Blocked nonregular two-level factorial designs. Technometrics, 46:269-279.

Chou, T.-C. (2006). Theoretical basis, experimental design, and computerized simulation of synergism and antagonism in drug combination studies. Pharmacological Reviews, 58:621-681.

Click, T. H., Liu, A., and Kaminski, G. A. (2011). Quality of random number generators significantly affects results of Monte Carlo simulations for organic and biological systems. Journal of Computational Chemistry, 32:513-524.

Deng, L.-Y. and Tang, B. (1999). Generalized resolution and minimum aberration criteria for Plackett-Burman and other nonregular factorial designs. Statistica Sinica, 9:10711082. 
Deng, L.-Y. and Tang, B. (2002). Design selection and classification for Hadamard matrices using generalized minimum aberration criteria. Technometrics, 44:173-184.

Devita, V. T., Young, R. C., and Canellos, G. P. (1975). Combination versus single agent chemotherapy: a review of the basis for selection of drug treatment of cancer. Cancer, $35: 98-110$.

Eendebak, P. T. and Schoen, E. D. (2017). Two-level designs to estimate all main effects and two-factor interactions. Technometrics, 59:69-79.

Fries, A. and Hunter, W. G. (1980). Minimum aberration $2^{k-p}$ designs. Technometrics, 22:601-608.

Garroi, J. J., Goos, P., and Sörensen, K. (2009). A variable-neighbourhood search algorithm for finding optimal run orders in the presence of serial correlation. Journal of Statistical Planning and Inference, 139:30-44.

Hansen, P. and Mladenović, N. (2001). Variable neighborhood search: Principles and applications. European Journal of Operational Research, 130:449-467.

Harvey, R. and Verseghy, D. L. (2016). The reliability of single precision computations in the simulation of deep soil heat diffusion in a land surface model. Climate Dinamics, $46: 3865-3882$.

He, Y. and Ding, C. H. Q. (2001). Using accurate arithmetics to improve numerical reproducibility and stability in parallel applications. The Journal of Supercomputing, 18:259277.

Hedayat, A., Sloane, N., and Stufken, J. (1999). Orthogonal Arrays: Theory and Applications. Springer.

John, P. W. M. (1961). Three-quarter replicates of $2^{4}$ and $2^{5}$ designs. Biometrics, 17:319321.

John, P. W. M. (1962). Three-quarter replicates of $2^{n}$ designs. Biometrics, 18:172-184. 
Lefebvre, S., Roblin, A., Varet, S., and Durand, G. (2010a). Metamodeling of aircraft infrared signature dispersion. Advance Statistical Analysis, 94:405-422.

Lefebvre, S., Roblin, A., Varet, S., and Durand, G. (2010b). A methodological approach for statistical evaluation of aircraft infrared signature. Reliability Engineering and System Safety, 95:484-493.

Li, W. and Lin, D. K. J. (2016). A note on foldover of $2^{k-p}$ designs with column permutations. Technometrics, 58:508-512.

Liao, C. T., Iyer, H. K., and Vecchia, D. F. (1996). Construction of orthogonal two-level designs of user specified resolution where $N \neq 2^{k}$. Technometrics, 38:342-353.

Lin, C. D. and Sitter, R. R. (2008). An isomorphism check for two-level fractional factorial designs. Journal of Statistical Planning and Inference, 138:1085-1101.

Mee, R. W. (2004). Efficient two-level designs for estimating all main effects and two-factor interactions. Journal of Quality Technology, 36:400-412.

Mee, R. W. (2009). A Comprehensive Guide to Factorial Two-Level Experimentation. Springer.

Mee, R. W. (2017). Generators for nonregular $2^{k-p}$ designs. Statistica Sinica, 27:1347-1369.

Meyer, R. K. and Nachtsheim, C. J. (1995). The coordinate-exchange algorithm for constructing exact optimal experimental designs. Technometrics, 37:60-69.

Miller, A. and Sitter, R. R. (2001). Using the folded-over 12-run Plackett-Burman design to consider interactions. Technometrics, 43:44-55.

Mukerjee, R. and Wu, C. F. J. (2006). A Modern Theory of Factorial Design. Springer.

Ning, S., Xu, H., Al-Shyoukh, I., Feng, J., and Sun, R. (2014). An application of a hill-based response surface model for a drug combination experiment on lung cancer. Statistics in Medicine, 33:4227-4236.

Pajak, T. F. and Addelman, S. (1975). Minimum full sequences of $2^{n-m}$ resolution III plans. Journal of the Royal Statistical Society Series B, 37:88-95. 
Ryan, K. J. and Bulutoglu, D. A. (2010). Minimum aberration fractional factorial designs with large N. Technometrics, 52:250-255.

Sartono, B., Goos, P., and Schoen, E. D. (2015). Constructing general orthogonal fractional factorial split-plot designs. Technometrics, 57:488-502.

Schoen, E. D. (2010). Optimum designs versus orthogonal arrays for main effects and two-factor interactions. Journal of Quality Technology, 42:197-208.

Schoen, E. D., Eendebak, P. T., and Nguyen, M. V. M. (2010). Complete enumeration of pure-level and mixed-level orthogonal arrays. Journal of Combinatorial Designs, 18:123140.

Straetemans, R., O’Brien, T., Wouters, L., Van Dun, J., Janicot, M., Bijnens, L., Burzykowski, T., and Aerts, M. (2005). Design and analysis of drug combination experiments. Biometrical Journal, 47:299-308.

Syafitri, U. and Goos, P. (2015). I-optimal design of mixture experiments in the presence of ingredient availability constraints. Journal of Quality Technology, 47:220-234.

Tang, B. and Deng, L.-Y. (1999). Minimum $G_{2}$-aberration for nonregular fractional factorial designs. The Annals of Statistics, 27:1914-1926.

Wu, C. F. J. and Hamada, M. S. (2009). Experiments: Planning, Analysis, and Optimization. Wiley.

Xiao, Q. and Xu, H. (2017). Construction of maximin distance Latin squares and related Latin hypercube designs. Biometrika, 104:455-464.

$\mathrm{Xu}$, H. (2009). Algorithmic construction of efficient fractional factorial designs with large run sizes. Technometrics, 51:262-277.

Xu, H. (2015). Nonregular factorial and supersaturated designs. Handbook of Design and Analysis of Experiments, pages 339-370.

Xu, H. and Wong, A. (2007). Two-level nonregular designs from quaternary linear codes. Statistica Sinica, 17:1191-1213. 
Table 1: Selected nonregular designs obtained from 32-run MA designs. $d$ : number of parent designs; ${ }^{s}$ : design permits the estimation of all $2 \mathrm{FIs} ;{ }^{r}$ : design has repeat runs.

\begin{tabular}{clllll}
\hline Factors & Runs & GR & $F_{4}(96,64,32)$ & $B_{4}$ & $d$ \\
\hline 9 & $96^{s r}$ & 4.66 & $(0,0,18)$ & 2 & 3 \\
10 & $96^{s r}$ & 4.66 & $(0,0,30)$ & 3.33 & 3 \\
& $160^{s r}$ & 4.8 & $(0,0,50)$ & 2 & 5 \\
\multirow{2}{*}{11} & 96 & 4.66 & $(0,0,69)$ & 7.67 & 3 \\
& $160^{s r}$ & 4.8 & $(0,0,113)$ & 4.52 & 5 \\
12 & 96 & 4.66 & $(0,0,108)$ & 12 & 3 \\
& $160^{s r}$ & 4.8 & $(0,0,178)$ & 7.12 & 5 \\
13 & 96 & 4.66 & $(0,0,155)$ & 17.22 & 3 \\
& $160^{r}$ & 4.8 & $(0,0,255)$ & 10.2 & 5 \\
14 & 96 & 4.66 & $(0,0,213)$ & 23.67 & 3 \\
& $160^{r}$ & 4.8 & $(0,0,349)$ & 13.96 & 5 \\
\multirow{2}{*}{15} & 96 & 4 & $(1,0,284)$ & 32.55 & 3 \\
& 160 & 4.4 & $(1,0,464)$ & 18.92 & 5 \\
& 96 & 4 & $(3,0,367)$ & 43.7 & 3 \\
& 128 & 4.5 & $(0,13,460)$ & 32 & 4 \\
& 160 & 4.4 & $(3,0,597)$ & 24.96 & 5 \\
\hline \multirow{2}{*}{16} & & & & &
\end{tabular}


Table 2: Selected nonregular designs obtained from 64-run MA designs. A dash as an element of the $F_{4}$ vector means that the corresponding $J_{4}$-characteristic does not exist. $d$ : number of parent designs.

\begin{tabular}{|c|c|c|c|c|c|}
\hline Factors & Runs & GR & $F_{4}(256,192,128,64)$ & $B_{4}$ & $d$ \\
\hline 17 & 192 & 4.66 & $(-, 0,0,153)$ & 17 & 3 \\
\hline 18 & 192 & 4.66 & $(-, 0,0,198)$ & 22 & 3 \\
\hline 19 & 192 & 4 & $(-, 1,0,243)$ & 28 & 3 \\
\hline \multirow[t]{3}{*}{20} & 192 & 4 & $(-, 2,0,291)$ & 34.33 & 3 \\
\hline & 256 & 4.5 & $(0,0,14,368)$ & 26.5 & 4 \\
\hline & 320 & 4.4 & $(0,2,0,459)$ & 19.08 & 5 \\
\hline \multirow[t]{3}{*}{21} & 192 & 4 & $(-, 7,0,483)$ & 60.66 & 3 \\
\hline & 256 & 4.5 & $(0,0,31,572)$ & 43.5 & 4 \\
\hline & 320 & 4.4 & $(0,7,0,769)$ & 33.28 & 5 \\
\hline \multirow[t]{3}{*}{22} & 192 & 4 & $(-, 11,0,579)$ & 75.33 & 3 \\
\hline & 256 & 4 & $(1,0,42,680)$ & 54 & 4 \\
\hline & 320 & 4.4 & $(0,13,0,917)$ & 41.36 & 5 \\
\hline \multirow[t]{3}{*}{23} & 192 & 4 & $(-, 15,0,685)$ & 91.11 & 3 \\
\hline & 256 & 4 & $(2,0,55,792)$ & 65.25 & 4 \\
\hline & 320 & 4.4 & $(0,19,0,1077)$ & 49.92 & 5 \\
\hline \multirow[t]{3}{*}{24} & 192 & 4 & $(-, 21,0,806)$ & 110.55 & 3 \\
\hline & 256 & 4 & $(4,0,71,924)$ & 79.5 & 4 \\
\hline & 320 & 4.4 & $(0,30,0,1267)$ & 61.48 & 5 \\
\hline \multirow[t]{3}{*}{25} & 192 & 4 & $(-, 26,0,949)$ & 131.44 & 3 \\
\hline & 256 & 4 & $(6,0,88,1080)$ & 95.5 & 4 \\
\hline & 320 & 4.4 & $(0,40,0,1475)$ & 73.4 & 5 \\
\hline \multirow[t]{3}{*}{26} & 192 & 4 & $(-, 38,0,1097)$ & 159.88 & 3 \\
\hline & 256 & 4 & $(11,0,108,1240)$ & 115.5 & 4 \\
\hline & 320 & 4.4 & $(0,63,0,1692)$ & 90.36 & 5 \\
\hline
\end{tabular}


Table 4: Selected nonregular designs obtained from 256-run MA designs. $d$ : number of parent designs; ${ }^{s}$ : design permits the estimation of all $2 \mathrm{FIs} ;{ }^{r}$ : design has repeat runs.

\begin{tabular}{|c|c|c|c|c|c|}
\hline Factors & Runs & GR & $F_{4}(768,512,256)$ & $B_{4}$ & $d$ \\
\hline 24 & $768^{s r}$ & 4.66 & $(0,0,66)$ & 7.33 & 3 \\
\hline 25 & $768^{s}$ & 4.66 & $(0,0,84)$ & 9.33 & 3 \\
\hline 26 & $768^{s}$ & 4.66 & $(0,0,105)$ & 11.67 & 3 \\
\hline 27 & $768^{s}$ & 4.66 & $(0,0,127)$ & 14.11 & 3 \\
\hline 28 & $768^{s}$ & 4.66 & $(0,0,152)$ & 16.88 & 3 \\
\hline 29 & $768^{s}$ & 4.66 & $(0,0,184)$ & 20.44 & 3 \\
\hline 30 & 768 & 4.66 & $(0,0,215)$ & 23.88 & 3 \\
\hline 31 & 768 & 4.66 & $(0,0,255)$ & 28.33 & 3 \\
\hline 32 & 768 & 4.66 & $(0,0,297)$ & 33 & 3 \\
\hline 33 & 768 & 4.66 & $(0,0,347)$ & 38.55 & 3 \\
\hline \multirow[t]{3}{*}{34} & 768 & 4.66 & $(0,0,404)$ & 44.88 & 3 \\
\hline & $1024^{s}$ & 4.75 & $(0,0,456)$ & 28.5 & 4 \\
\hline & $1280^{s}$ & 4.8 & $(0,0,608)$ & 24.32 & 5 \\
\hline \multirow[t]{3}{*}{35} & 768 & 4 & $(1,0,455)$ & 51.55 & 3 \\
\hline & $1024^{s}$ & 4.5 & $(0,10,496)$ & 33.5 & 4 \\
\hline & $1280^{s}$ & 4.8 & $(0,0,692)$ & 27.68 & 5 \\
\hline \multirow[t]{3}{*}{36} & 768 & 4 & $(3,0,492)$ & 57.66 & 3 \\
\hline & $1024^{s}$ & 4.5 & $(0,19,560)$ & 39.75 & 4 \\
\hline & $1280^{s}$ & 4.8 & $(0,0,773)$ & 30.92 & 5 \\
\hline
\end{tabular}

Table 5: Strength-3 design options for the 28-factor infrared sensor experiment.

\begin{tabular}{lllccc}
\hline Runs & GR & $F_{4}(256,128)$ & $B_{4}$ & D-efficiency $(\%)$ & Blocks \\
\hline \multirow{2}{*}{768} & 4.66 & $(152,0)$ & 16.88 & 83.38 & 3 \\
& 4.66 & $(25,870)$ & 26.94 & 74.61 & 6 \\
896 & 4.857 & $(0,1080)$ & 22.04 & 79.90 & 7 \\
\hline
\end{tabular}

